\title{
PRUEBA DE LA TEORÍA DEL INTERCAMBIO Y LA TEORÍA DEL ORDEN JERÁRQUICO DE LA ESTRUCTURA DE CAPITAL, EVIDENCIA ECUATORIANA
}

\section{TESTING THE TRADE-OFF THEORY AND THE PECKING ORDER THEORY OF CAPITAL STRUCTURE, ECUADORIAN EVIDENCE}

\author{
Diana Castro Herrera \\ Christian Morán Montalvo \\ Leslie Rodríguez Valencia
}

Facultad de Economía y Ciencias Empresariales, Universidad Espíritu Santo, Km. 2.5 Vía La Puntilla, Samborondón-Ecuador. cmoranm@uees.edu.ec

\section{RESUMEN}

Este artículo investiga si las compañías industriales, que cotizaron en la bolsa de valores de Guayaquil durante el periodo 2011 hasta 2015, basaron su estructura de capital en las teorías del Intercambio y del Orden Jerárquico. Se escogió el segmento alimentos y bebidas el cual fue dividido en: compañías que procesan alimentos y compañías que venden productos básicos. Se estudiaron 11 empresas. Para analizar la primera teoría, la estructura capital óptima fue determinada. Las compañías que procesan alimentos tienden a estar sub-endeudadas mientras que las compañías que venden productos básicos están sobre-endeudadas. Para analizar la segunda teoría, el orden de preferencia para financiar los activos fue evaluado, como: en primer lugar, sus recursos internos, luego deuda y finalmente capital. Adicional, fue considerado el monto de las utilidades acumuladas de cada empresa. La mayoría de las empresas evaluadas prefieren emitir deuda. Además, las empresas fueron valoradas mediante el Flujo de efectivo descontado. Se encontró que las compañías en estudio no utilizan las teorías mencionadas. Estas utilizan las políticas de la Bolsa de Valores de Guayaquil y las propias de cada empresa. Sin embargo, se sugiere que los gerentes las consideren con el fin de optimizar el valor de la empresa y reducir costos.

Palabras Claves: Teoría del Intercambio, Teoría del Orden Jerárquico, beta, endeudamiento, costo de capital.

\begin{abstract}
This paper investigates if industrial companies, especially food \& beverage divided into food processing and raw materials, listed on the Guayaquil Stock Exchange from 2011 to 2015 chose their capital structure based on the Trade-off theory and the Pecking Order Theory. The sample consisted of 11 companies. For the trade-off theory, the optimal capital structure was discovered. Food processing companies tend to be underleveraged whereas raw material companies are overleveraged. For the Pecking order theory, the order of preference for financing their assets was evaluated such as: first of all, retained earnings after that, debt and finally, equity. In addition, their retained earnings were considered. Most of the companies preferred to issue debt. The free-cash-flow method was used for this study in order to discover company's value. Analyzed companies did not consider both theories, instead they considered the Guayaquil Stock Exchange policy and their own policies. However, the studied theories must be taken into account in order to maximize company's value and to reduce costs.
\end{abstract}

Keywords: Trade-off theory, Pecking order theory, beta, leverage, cost of capital.

Recibido: 26/09/2017;

Aceptado: 10/11/2017;

Publicado: 19/12/2017 


\section{Introducción}

El debate de la Estructura de Capital comenzó con el teorema de Modigliani-Miller (1958) que demostró que las decisiones de la estructura de capital no eran importantes porque no influían en la valoración de la empresa. Sin embargo, estas decisiones afectan en gran medida la valoración del negocio ya que hay fricciones en el mercado como: impuestos, costos de quiebra, información asimétrica, etc.

Algunas teorías han sido desarrolladas como: Intercambio, Elección jerárquica, ánimo del mercado, costos de agencia, ciclo de vida, etc. Las teorías del intercambio y de la elección jerárquica son muy conocidas. Los investigadores han realizado gran cantidad de trabajo empírico con el fin de probar ambas teorías, (Jensen \& Uhl, 2008) [1], por ejemplo: El estudio de Rajan y Zingales. Sin embargo, no hay ninguna pista sobre qué teoría predomina y se ajusta a la realidad.

Por esta razón, sigue siendo un tema discutible y controvertido en Finanzas Corporativas. Por ejemplo, hay algunos autores que favorecen la teoría del Intercambio: (Chirinko \& Singha, 2000) [2] (Frank \& Goyal, 2003) [3], y (Leary \& Roberts, 2010) [4]. Mientras que (Shyam-Sunder \& Myers, 1999) [5] y (Lemmon \& Zender, 2010) [6] favorecen la teoría del Orden Jerárquico. (Miglo, 2014) [7].

La mayoría de los estudios se realizan en países desarrollados debido a la información disponible. Sin embargo, no existe un estudio relacionado con empresas ecuatorianas. El sector industrial fue elegido porque representó en 2014 , el $11,8 \%$ del Producto Interno Bruto (PIB), 100,54 millones de dólares. Un enfoque especial en el segmento de alimentos y bebidas, cuya participación fue del $38 \%$ de la industria manufacturera. Este segmento generó \$ 5.297 millones (Maldonado \& Proaño, 2015) [8]. La mayoría de las compañías en referencia emitieron deuda y solo una emitió acciones en la Bolsa de Valores de Guayaquil de 2011 a 2015.

A lo largo de este documento, se podrá ver si las empresas estudiadas eligieron su estructura de capital basada en las teorías del Intercambio 0 en la teoría del Orden Jerárquico. Esto daría una idea de cómo las empresas podrían mejorar sus decisiones para minimizar el costo del capital y optimizar su valor.

\section{Marco conceptual}

\subsection{La teoría del intercambio de la estructura de capital}

Hay dos formas en que las empresas pueden financiar sus activos: deuda o emisión de acciones. La ventaja de la primera: los ahorros fiscales de intereses (tax shield). Por lo tanto, hay un intercambio entre los ahorros fiscales de intereses y costo de la insolvencia financiera. Hay beneficios fiscales porque el interés (costo de la deuda) es deducible de impuestos. Un aumento de deuda podría ser beneficioso por el escudo fiscal, sin embargo, es importante considerar que mucha deuda lleva a la compañía a la insolvencia financiera, la cual es perjudicial.

Las razones de endeudamiento dependen de las empresas. Por ejemplo, se espera que las compañías que tengan activos tangibles, posean mayores razones de endeudamiento. Mientras que las empresas en riesgo, no rentables y con activos intangibles se financien con emisión de acciones (Brealey, Myers, Allen, 2011) [9]. "El óptimo teórico se alcanza cuando el valor presente de los ahorros en impuestos generados por prestamos adicionales queda cancelado por los incrementos en el valor presente de los costos de la insolvencia' (Brealey, Myers, Allen, 2011, p. 447) [10].

\subsection{Elección jerárquica de las opciones de financiamiento}

Donaldson (1961) observó que los gerentes financieros prefieren financiar sus inversiones mediante recursos internos en lugar de recursos externos. Myers y Majluf (1984) trabajaron en esta teoría y escribieron un documento de Finanzas corporativas y decisiones de inversión cuando las empresas tienen información que los inversionistas no tienen. La idea general es que los gerentes están mejor informados que los inversionistas (Información Asimétrica). En otras palabras, los gerentes saben más sobre las perspectivas de la empresa. Por ejemplo, cuando la gerencia decide aumentar los dividendos, el precio por acción normalmente aumenta porque es una señal de que la empresa está bien. Esta información asimétrica influye en las decisiones de la estructura de capital.

En primer lugar, los gestores prefieren recaudar fondos mediante el financiamiento interno (utilidades acumuladas), luego de emitir deuda y finalmente emitir acciones como último recurso, cuando la capacidad de endeudamiento ha alcanzado su máximo y 
podría llevar a la quiebra. (Martin, H. Kent Baker and Gerald S., 2011) [11].

Se supone que la transmisión de información es costosa, y los administradores y los inversionistas son racionales. Son reacios a emitir capital cuando piensan que la acción está subvaluada. Por eso los inversionistas perciben la emisión de acciones como un factor que los gerentes creen que las acciones están sobrevaloradas. Por lo tanto, el precio de la acción cae. Debido a esta reacción negativa, las compañías deciden no emitir acciones y prefieren emitir deuda. (Grinblatt-Titman, 2002) [12].

Es importante mencionar que las empresas de alta tecnología prefieren emitir acciones, ya que no tienen activos tangibles que sirvan para garantizar la deuda. (Brealey, Myers, Allen, 2011).

\section{Materiales y métodos}

\subsection{Muestra de las compañías}

Con el objetivo de probar la Teoría del Intercambio y la Teoría de la Elección Jerárquica, se consideraron empresas industriales (alimentos y bebidas) puesto que estas representan $38 \%$ del PIB manufacturero. Para el presente artículo, se ha considerado dos segmentos: alimentos procesados y productos básicos.

La muestra consistió en 11 empresas en total por la disponibilidad de la información financiera. Ocho empresas procesadoras de alimentos y tres empresas de productos básicos. El tamaño de las empresas oscila entre $\$ 15$ millones y $\$ 137$ millones. El tamaño se mide por ventas anuales. Empresas con datos históricos y rentables han sido estudiadas.

Tabla 1. Tamaño empresas procesadoras de alimentos

En millones de dolares (USD)

\begin{tabular}{llc} 
Año & Companñía & Tamaño \\
\hline 2011 & Industrias Toni & 118.53 \\
2012 & Heladosa & 17.75 \\
2012 & Floralp & 16.48 \\
2013 & Industria Lojana de Epicerías & 21.38 \\
2014 & Universal Sweet Industries & 40.90 \\
2015 & Sumesa & 33.99 \\
2015 & Alimentsa & 74.94 \\
2015 & Moderna Alimentos & 137.89 \\
\hline
\end{tabular}

En millones de dolares (USD)

\begin{tabular}{llc} 
Año & Compañía & Tamaño \\
\hline 2013 & Compañía Azucarera Valdez & 133.38 \\
2014 & Cofina & 15.07 \\
2015 & Labiza & 17.75 \\
\hline
\end{tabular}

El periodo 2011 hasta 2015 fue elegido puesto que, en el 2011, Ecuador registró una tasa de crecimiento porcentual anual del PIB a precios constantes sobre la base de dólares de EE.UU. del 7.868\% (Banco Mundial, 2017) [13] y hasta el 2015 por la disponibilidad de la información cuando se efectuó el estudio.

\subsection{Tipos de investigación}

Investigación descriptiva: este artículo describe como se realizó la investigación de acuerdo con las teorías estudiadas y el modelo de valoración.

\subsection{Fuente}

Cada empresa se analizó en base a su reporte anual y reporte de riesgo los cuales fueron obtenidos en la Bolsa de Valores de Guayaquil.

\subsection{Valoración de la empresa}

Los estados financieros (Estado de Situación Financiera, Estado de Resultados y Flujo de Caja) y el reporte anual fueron utilizados para realizar un plan de negocios de cinco años con el objetivo de determinar los flujos de efectivo descontados. Se utilizó la fórmula siguiente:

$F C F=E B I T(1-T)+D-N C E-\triangle W C$

Donde :

$\checkmark \quad$ FCF: Flujo de efectivo descontado

$\checkmark$ EBIT: Earnings before interests and taxes, ganancias antes de intereses e impuestos

$\checkmark \quad$ T: Impuestos. El impuesto corporativo en Ecuador es del 25\%, sin embargo, este ha bajado gradualmente 1\% desde 2011 hasta 2013 con el fin de alcanzar el $22 \%$ (Asamblea Nacional Ecuador, 2010) [14]. Adicional, se debe considerar el $15 \%$ a la utilidad trabajadores. Por lo tanto, el impuesto corporativo en Ecuador es del $34.21 \%$ en promedio.

$\checkmark$ D: Depreciación

$\checkmark$ NCE: Gasto neto de capital

WC: Variación de Capital de trabajo

Tabla 2. Tamaño empresas productos básicos 
Finalmente, se estima el valor terminal porque se espera que la firma opere en el largo plazo. La fórmula 2 es usada:

$$
V T E_{n=\frac{C F_{n+1}}{k-g_{2}}}
$$

Donde :

$\checkmark C F_{n+1}$ : El flujo de efectivo posterior al plan de negocio crece $\left(g_{2}\right)$

$\checkmark$ K: costo de capital

$\checkmark g_{2 .}$ : se asume que las empresas tendrán un crecimiento perpetuo del $1 \%$ por razones de simplicidad.

Los flujos de efectivo fueron descontados a través del costo promedio ponderado de capital (WACC). El costo promedio ponderado de capital fue calculado usando el modelo de valoración de activos fijos (CAPM). El modelo de valoración de activos fijos usa los elementos siguientes:

$E(R)=R_{f}+B\left[E\left(R_{M}\right)-\left(R_{f}\right)\right]+C$

Donde :

$\checkmark$ Tasa libre de riesgo $\left(R_{f}\right)$ : Bonos del tesoro de Estados Unidos.

$\checkmark$ Beta $(B)$ : sector alimentos, economías emergente tomado del sitio web Damodaran.

$\checkmark$ Prima de riesgo $\left(R_{M}\right)$ : Prima de riesgo histórica Estados Unidos.

Cuando el país es riesgoso, se puede agregar una prima de riesgo como el riesgo país (C). Fuente: Banco Central del Ecuador.

El costo de capital se calculó utilizando la fórmula 3. Beta contiene información del sector como: apalancamiento $\left(L_{s}\right)$ e impuestos $\left(T_{s}\right)$. Por esta razón, es necesario desapalancar las betas con el fin de aplicar el apalancamiento e impuestos de la empresa. La fórmula 4 fue utilizada:

$$
B_{a s}=\frac{B_{c s}+B_{d s} L_{s}(1-T)}{1+L_{s}(1-T)}
$$

Donde:

$$
\begin{array}{ll}
\checkmark & \text { Beta del sector }\left(B_{c s}\right) \\
\checkmark & \text { Beta de la deuda del sector }\left(B_{d s}\right) \\
\checkmark & \text { Apalancamiento del sector }\left(L_{s}\right) \\
\checkmark & \text { Impuestos }(T)
\end{array}
$$

La fórmula Hamada (1969), calcula la beta del capital de la compañía. En otras palabras, el impacto de la deuda de la compañía debe ser adicionada a la beta desapalancada.

$$
B_{c j}=B_{A s}+\left(B_{A s}-B_{d j}\right) L_{j}\left(1-T_{c}\right)
$$

Donde:

$\checkmark$ Beta capital de la empresa $\left(B_{c j}\right)$

$\checkmark$ Beta desapalancada del sector $\left(B_{A s}\right)$

$\checkmark$ Beta de la deuda de la empresa $\left(B_{d j}\right)$

$\checkmark$ Apalancamiento de la firma $\left(L_{j}\right)$

$\checkmark$ Impuesto corporativo $\left(T_{c}\right)$

Se explica el cálculo del costo de la deuda a través de las fórmulas 7 hasta 14. EI WACC considera el costo de capital y el costo de deuda. La fórmula del WACC es la siguiente:

$$
W A C C=r d\left(\frac{D}{D+E}\right)\left(1-T_{c}\right)+r c\left(\frac{E}{D+E}(6)\right.
$$

Donde:

$\checkmark$ Costo de deuda $(r d)$

$\checkmark$ Porcentaje de financiamiento con deuda $(\mathrm{D} / \mathrm{D}+\mathrm{E})$

$\checkmark$ Impuesto corporativo $\left(T_{c}\right)$

$\checkmark$ Costo de capital ( $r c$ )

$\checkmark$ Porcentaje de financiamiento con capital $(\mathrm{E} / \mathrm{D}+\mathrm{E})$

Finalmente, para encontrar el valor del capital, la deuda neta tiene que ser sustraída. La deuda neta consiste en deuda financiera a corto y largo plazo menos el efectivo. La deuda neta permite medir si la empresa tiene la capacidad de pagar sus deudas en la actualidad.

\subsection{Analizando las teorías}

\subsubsection{La teoría del intercambio}

Para esta teoría, la estructura de capital óptima fue encontrada. Los cálculos se basaron en la hoja de cálculo de Damodaran [15]. Sin embargo, ésta fue adaptada a la información disponible. El valor en libros fue considerado para los cálculos.

La información considerada para el análisis es la siguiente: ganancias antes de intereses, depreciación y amortización (EBITDA), depreciación y amortización, gasto capital, intereses, impuesto, costo de deuda antes de impuestos, valor contable de capital, beta, valor contable de la deuda, tasa libre de riesgo, prima de riesgo y riesgo país. 
Se crearon varios escenarios con diferentes niveles de deuda de $0 \%$ a $90 \%$. El costo de capital (rc) fue calculado mediante la fórmula 3. La beta cambia cuando el apalancamiento incrementa. Para calcular ese cambio, la fórmula 5 fue utilizada.

El costo de deuda sigue un tratamiento especial. Primero se utiliza la fórmula 7:

$$
\text { Razón de deuda }=\frac{\text { Deuda }}{\text { Deuda }+ \text { Capital }}
$$

Ambos valores (deuda y capital) son tomados del Estado de Situación Financiera.

El apalancamiento es calculado:

$$
\text { Apalancamiento }=\frac{\text { Razón de deuda }}{(1-\text { razón de deuda })}
$$

Para comprobar la deuda

$$
\text { deuda }=\text { razón de deuda } *(D+E)
$$

Donde:

$\checkmark$ D: la deuda es considerada a partir del Estado de Situación Financiera

$\checkmark$ E: capital es tomado a partir del Estado de Situación Financiera

Intereses

Si la razón de deuda es menor que la razón de deuda del escenario (columna de la tabla 3 ), los intereses se calculan de la manera siguiente:

$$
\text { Intereses }=\frac{\text { intereses }(\text { gasto })}{\text { valor en libros deuda }}
$$

Los intereses y el valor en libros de la deuda fueron tomados a partir del estado de resultados y del estado de situación financiera, respectivamente.

Si la razón de deuda es superior a la razón de deuda del escenario, los intereses son calculados de la manera siguiente:

$$
\text { Intereses }=\text { intereses }(\text { gasto })+r d^{*}(D R S-C D R)
$$

\section{Donde}

$\checkmark$ Gasto intereses actuales

$\checkmark$ Costo de deuda antes de impuestos (rd)

$\checkmark$ Razón de deuda del escenario (DRS)

$\checkmark$ Razón deuda (CDR)
La renta imponible es calculada:

$$
\text { Renta imponible }=\text { EBIT }- \text { intereses }
$$

El EBIT es constante en todos los diferentes niveles de deuda.

Tabla 3. Desglose del costo de deuda

En millones de dólares (USD)

\begin{tabular}{ccccc}
$\mathrm{D} /(\mathrm{D}+\mathrm{E})$ & $\begin{array}{c}\text { Apalancamie } \\
\text { nto }\end{array}$ & Deuda & Interes & $\begin{array}{c}\text { Renta } \\
\text { imponibl } \\
\mathrm{e}\end{array}$ \\
\hline $0 \%$ & - & 0.00 & 0.00 & 29.68 \\
$10 \%$ & 0.11 & 12.53 & 0.38 & 29.30 \\
$20 \%$ & 0.25 & 25.06 & 0.77 & 28.91 \\
$30 \%$ & 0.43 & 37.59 & 1.15 & 28.53 \\
$40 \%$ & 0.67 & 50.12 & 1.54 & 28.14 \\
$50 \%$ & 1.00 & 62.65 & 2.00 & 27.69 \\
$60 \%$ & 1.50 & 75.18 & 3.59 & 26.09 \\
$70 \%$ & 2.33 & 87.71 & 5.35 & 24.33 \\
$80 \%$ & 4.00 & 100.24 & 7.22 & 22.46 \\
$90 \%$ & 9.00 & 112.77 & 10.61 & 19.07 \\
\hline
\end{tabular}

Después de esto, se determina el coeficiente de cobertura de intereses antes de impuestos (ICR):

$I C R=\frac{\text { Renta imponible }}{\text { Intereses }}$

Este coeficiente de cobertura de intereses

\begin{tabular}{|c|c|c|c|c|}
\hline $\begin{array}{c}\mathrm{D} /(\mathrm{D}+\mathrm{E} \\
)\end{array}$ & ICR & $\begin{array}{c}\text { Calificació } \\
n\end{array}$ & $\begin{array}{c}\text { Antes } \\
\text { impuesto } \\
\text { s rj }\end{array}$ & rj \\
\hline $0 \%$ & $\begin{array}{c}\infty \\
77.2\end{array}$ & Aaa/AAA & $12.37 \%$ & $8.20 \%$ \\
\hline $10 \%$ & $\begin{array}{c}6 \\
38.6\end{array}$ & Aaa/AAA & $12.37 \%$ & $8.20 \%$ \\
\hline $20 \%$ & $\begin{array}{c}3 \\
25.7\end{array}$ & Aaa/AAA & $12.37 \%$ & $8.20 \%$ \\
\hline $30 \%$ & $\begin{array}{c}5 \\
19.3\end{array}$ & Aaa/AAA & $12.37 \%$ & $8.20 \%$ \\
\hline $40 \%$ & $\begin{array}{c}2 \\
14.8\end{array}$ & Aaa/AAA & $12.37 \%$ & $8.20 \%$ \\
\hline $50 \%$ & 7 & Aaa/AAA & $12.37 \%$ & $8.20 \%$ \\
\hline $60 \%$ & 8.26 & $\mathrm{~A} 1 / \mathrm{A}+$ & $12.72 \%$ & $8.43 \%$ \\
\hline $70 \%$ & 5.54 & $\mathrm{~A} 3 / \mathrm{A}-$ & $13.37 \%$ & $8.86 \%$ \\
\hline $80 \%$ & 4.11 & Baa2/BBB & $13.87 \%$ & $9.19 \%$ \\
\hline $90 \%$ & 2.80 & $\mathrm{~B} 1 / \mathrm{B}+$ & $17.12 \%$ & $\begin{array}{c}11.35 \\
\%\end{array}$ \\
\hline
\end{tabular}
antes de impuestos sirve para estimar una calificación sintética para las empresas pequeñas y con mayor riesgo (Aswath Damodaran, n.d.) [16].

Tabla 4. Desglose del costo de deuda, parte 2 
La Tabla 4 muestra que el escenario de razón de deuda de $0 \%$ a $50 \%$ es lo suficientemente bueno para obtener una calificación de Aaa / AAA. Por lo tanto, el costo antes de impuestos de la deuda es el mismo. Después de este punto, la tasa de cobertura de intereses antes de impuestos es menor, como resultado, la calificación disminuye. El costo antes de impuestos de la deuda se calcula utilizando el diferencial de la calificación, más la tasa libre de riesgo y el riesgo país. Finalmente, el costo de la deuda se calcula de la manera siguiente:

$r j=\operatorname{Pre}-\operatorname{tax} r j *(1-T)$

\section{Donde}

$\checkmark$ Costo de la deuda después de impuestos(rj)

$\checkmark$ Costo de la deuda antes de impuestos (Pre-tax rj)

$\checkmark$ Tasa corporativa de impuestos (T)

La Tabla 5 muestra a medida que el nivel de deuda incrementa, el costo de capital sigue la misma dirección puesto que los accionistas requerirán una prima por el riesgo extra. El costo de deuda incrementa también. Aquí un ejemplo:

Tabla 5: Financiación óptima En millones de dólares (USD)*

\begin{tabular}{ccccc}
\hline $\mathrm{D} /(\mathrm{D}+\mathrm{E})$ & $\mathrm{rc}$ & $\mathrm{rd}$ & WACC & $\begin{array}{c}\text { Valor de la } \\
\text { Empresa* }^{*}\end{array}$ \\
\hline $0 \%$ & $14 \%$ & $8.20 \%$ & $14.25 \%$ & 40.87 \\
$10 \%$ & $14 \%$ & $8.20 \%$ & $13.82 \%$ & 42 \\
$20 \%$ & $15 \%$ & $8.20 \%$ & $13.39 \%$ & 43.2 \\
$30 \%$ & $15 \%$ & $8.20 \%$ & $12.95 \%$ & 44.47 \\
$40 \%$ & $15 \%$ & $8.20 \%$ & $12.52 \%$ & 45.83 \\
$50 \%$ & $16 \%$ & $8.20 \%$ & $12.09 \%$ & 47.29 \\
$60 \%$ & $17 \%$ & $8.43 \%$ & $11.80 \%$ & 48.33 \\
$70 \%$ & $18 \%$ & $8.86 \%$ & $11.70 \%$ & 48.72 \\
$\mathbf{8 0} \%$ & $\mathbf{2 1} \%$ & $\mathbf{9 . 1 9 \%}$ & $\mathbf{1 1 . 6 0} \%$ & $\mathbf{4 9 . 0 9}$ \\
$90 \%$ & $30 \%$ & $11.35 \%$ & $13.21 \%$ & 43.72 \\
\hline
\end{tabular}

Esta tabla muestra, a medida que sube la razón de deuda, el WACC disminuye hasta $11.60 \%$, el cual es el mínimo. Esto se debe por el escudo fiscal de intereses, aunque el costo de capital y el costo de deuda aumentan. Después de eso, subirá porque el escudo fiscal de intereses no proporciona esa ventaja puesto que existe el riesgo de quiebra. Eso es coherente con la teoría del intercambio. Con el escenario de razón deuda, $80 \%$, la compañía maximiza su valor. Para calcular el valor de la empresa, por favor revisar el punto 3.4 evaluación de la empresa.

\subsubsection{Teoría de la elección jerárquica}

La metodología para probar esta teoría fue analizar cómo las empresas prefieren financiar sus activos. Las utilidades retenidas de cada empresa se tomaron del estado de resultados.

\section{Resultados}

No hay evidencia de que la muestra de empresas escoja su estructura de capital de acuerdo con las teorías analizadas. Las empresas eligieron su estructura de capital de acuerdo con la política de la bolsa de valores ecuatoriana. Por ejemplo, el importe de la emisión de bonos no tiene que ser superior al $80 \%$ del importe total de los activos no comprometidos. (Consejo Nacional de Valores, 2015) [17]. Las empresas también tienen su propia política. Sin embargo, los gerentes deben considerar estas teorías al momento de tomar decisiones.

\subsection{La teoría del intercambio}

El análisis de la teoría del intercambio lleva a cabo la estructura de capital óptima. Se espera que las empresas estudiadas tengan altas razones de deuda ya que tienen activos tangibles. Sin embargo, se encontró que las empresas que procesan alimentos usualmente están un poco sub-endeudadas. Mientras que las compañías que venden productos básicos estas sobre-endeudadas.

Tabla 6. Comparación razón deuda, empresas que

\begin{tabular}{lccc}
\multicolumn{4}{c}{ procesan alimentos } \\
\hline Compañía & $\begin{array}{l}\text { Razón } \\
\text { deuda } \\
\text { actual }\end{array}$ & $\begin{array}{c}\text { Razón } \\
\text { deuda } \\
\text { óptima }\end{array}$ & $(\Delta)$ \\
\hline Industrias Toni & $56.56 \%$ & $60 \%$ & $\begin{array}{c}3.44 \% \\
-\end{array}$ \\
Heladosa & $51.28 \%$ & $50 \%$ & $1.28 \%$ \\
Floralp & $60.51 \%$ & $60 \%$ & $0.51 \%$ \\
Industria lojana & $63.61 \%$ & $70 \%$ & $6.39 \%$ \\
Universal sweet & $67.56 \%$ & $70 \%$ & $2.44 \%$ \\
Sumesa & $70.00 \%$ & $60 \%$ & $-10 \%$ \\
Alimentsa & $55.67 \%$ & $90 \%$ & 34.33 \\
Moderna & $49.36 \%$ & $80 \%$ & 30.64 \\
$\%$
\end{tabular}

La razón de deuda actual se calculó utilizando la fórmula 7. La razón de deuda óptima, véase la teoría del intercambio de este artículo que 
está en la sección 3.5.1. La última columna de la tabla 6 se refiere a la brecha entre la razón de deuda óptima y el coeficiente de deuda actual.

Tabla 7. WACC-Empresas que procesan alimentos

\begin{tabular}{|c|c|c|c|}
\hline Compañía & $\begin{array}{l}\text { Razón } \\
\text { deuda } \\
\text { actual }\end{array}$ & $\begin{array}{l}\text { Razón } \\
\text { deuda } \\
\text { óptima }\end{array}$ & (A) \\
\hline Industrias Toni & $\begin{array}{c}11.74 \\
\%\end{array}$ & $11.68 \%$ & $-0.07 \%$ \\
\hline Heladosa & $\begin{array}{c}11.92 \\
\%\end{array}$ & $10.96 \%$ & $-0.96 \%$ \\
\hline Floralp & $\begin{array}{c}12.04 \\
\%\end{array}$ & $10.69 \%$ & $-1.35 \%$ \\
\hline Industria Lojana & $7.42 \%$ & $7.40 \%$ & $-0.03 \%$ \\
\hline Universal sweet & $8.04 \%$ & $8.01 \%$ & $-0.03 \%$ \\
\hline Sumesa & $\begin{array}{c}12.19 \\
\%\end{array}$ & $10.97 \%$ & $-1.22 \%$ \\
\hline Alimentsa & $\begin{array}{c}10.18 \\
\%\end{array}$ & $10.02 \%$ & $-0.17 \%$ \\
\hline Moderna & $\begin{array}{c}12,33 \\
\% \\
\end{array}$ & $11,60 \%$ & $-0,73 \%$ \\
\hline
\end{tabular}

La tabla 7 muestra el WACC con el coeficiente de deuda actual y el coeficiente de deuda óptima. EI WACC tiene que ser minimizado para maximizar el valor de la empresa. Para calcular WACC, consulte 3.5.1. la teoría del intercambio de este artículo (costo de capital y costo de la deuda). Finalmente se usó la fórmula 6.

Por su parte, la Tabla 8 muestra el valor de la empresa con el coeficiente de endeudamiento actual y la razón de deuda óptima.

Tabla 8. Valoración de las empresas que procesan alimentos

En millones de dólares (USD)

\begin{tabular}{cccc} 
Compañía & $\begin{array}{l}\text { Razón } \\
\text { deuda } \\
\text { actual }\end{array}$ & $\begin{array}{c}\text { Razón } \\
\text { deuda } \\
\text { óptima }\end{array}$ & \\
\hline Industrias Toni & 140.9 & 141.84 & 0.94 \\
Heladosa & 28.47 & 31.12 & 2.65 \\
Floralp & 6.49 & 7.52 & 1.03 \\
Industria Lojana & 14.69 & 14.75 & 0.05 \\
Universal sweet & 133.33 & 133.84 & 0.51 \\
Sumesa & 27.33 & 30.45 & 3.12 \\
Alimentsa & 60.51 & 61.63 & 1.11 \\
Moderna & 46,48 & 49,09 & 2,60 \\
\hline
\end{tabular}

Tabla 9. Comparación razón deuda, productos básicos

\begin{tabular}{cccc}
\hline Compañía & $\begin{array}{l}\text { Razón } \\
\text { deuda } \\
\text { actual }\end{array}$ & $\begin{array}{l}\text { Razón } \\
\text { deuda } \\
\text { óptima }\end{array}$ & (4) \\
\hline $\begin{array}{c}\text { Azucarera } \\
\text { Valdéz }\end{array}$ & $48.29 \%$ & $40 \%$ & $-8.29 \%$ \\
Cofina & $62.35 \%$ & $10 \%$ & $-52.35 \%$ \\
Labiza & $65.55 \%$ & $30 \%$ & $-35.55 \%$ \\
\hline
\end{tabular}

Tabla 10. WACC-Empresas productos básicos

\begin{tabular}{|c|c|c|c|}
\hline Compañía & $\begin{array}{l}\text { Razón } \\
\text { deuda } \\
\text { actual }\end{array}$ & $\begin{array}{l}\text { Razón } \\
\text { deuda } \\
\text { óptima }\end{array}$ & $(A)$ \\
\hline $\begin{array}{c}\text { Azucarera } \\
\text { Valdéz }\end{array}$ & $11.26 \%$ & $10.29 \%$ & $0.97 \%$ \\
\hline Labiza & $15.46 \%$ & $15.29 \%$ & $\begin{array}{c}- \\
0.17 \%\end{array}$ \\
\hline Cofina & $9.77 \%$ & $8.84 \%$ & $-\overline{-}$ \\
\hline
\end{tabular}

Tabla 11. Valoración de empresas de productos básicos

\begin{tabular}{|c|c|c|c|}
\hline Compañía & $\begin{array}{l}\text { Razón } \\
\text { deuda } \\
\text { actual }\end{array}$ & $\begin{array}{l}\text { Razón } \\
\text { deuda } \\
\text { óptima }\end{array}$ & $(\Delta)$ \\
\hline Azucarera Valdéz & 124.85 & 138.01 & $\begin{array}{c}13.15 \\
\%\end{array}$ \\
\hline Labiza & 21.74 & 21.99 & $0.25 \%$ \\
\hline Cofina & 12.94 & 14.32 & $1.38 \%$ \\
\hline
\end{tabular}

\section{Análisis}

\subsection{La teoría del intercambio}

La gran mayoría de las empresas que procesan alimentos se aproximan al coeficiente de deuda óptima, véase la tabla 6 , excepto Alimentsa y Moderna Alimentos. Las dos últimas empresas podrían aumentar su ratio de deuda en 30 puntos. Además de esto, a medida que la razón de la deuda aumenta, el costo del capital también aumenta porque los accionistas van a solicitar una mayor rentabilidad, ya que hay más riesgo. Sin embargo, esto es compensado por los escudos fiscales de intereses.

Es importante mencionar que estas empresas podrían aumentar su razón de deuda puesto que su coeficiente de cobertura de intereses es mayor. En otras palabras, el coeficiente de cobertura de intereses mide cuántas veces la compañía puede pagar los intereses de su deuda pendiente con su renta imponible 
Tabla 12. Razón cobertura intereses-

Compañías que procesan alimentos

\begin{tabular}{cc} 
Compañía & $\begin{array}{c}\text { Razón cobertura } \\
\text { intereses }\end{array}$ \\
\hline Industrias Toni & 6.74 \\
Heladosa & 4.99 \\
Floralp & 5.64 \\
Industria Lojana de Epicerías & 10.47 \\
Universal sweet industries & 5.49 \\
Sumesa & 3.74 \\
Alimentsa & 18.16 \\
Moderna Alimentos & 15.65 \\
\hline
\end{tabular}

Alimentsa y Moderna Alimentos tienen una proporción significativa de cobertura de intereses. Por lo tanto, podrían aumentar su razón de deuda para maximizar el valor de la compañía.

Tabla 13. Razón cobertura intereses-productos básicos

\begin{tabular}{cc}
\hline \multicolumn{2}{c}{ básicos } \\
Compañía & $\begin{array}{c}\text { Razón cobertura } \\
\text { intereses }\end{array}$ \\
Azucarera Valdez & 3.66 \\
Cofina & 0.85 \\
Labiza & 1.97 \\
\hline
\end{tabular}

Las empresas de productos básicos tienen una tasa de cobertura de interés baja. Por lo tanto, deben bajar su coeficiente de deuda para maximizar el valor de la empresa.

\subsection{Teoría de la elección jerárquica}

La mayoría de las empresas no financian sus inversiones ni reestructuran sus deudas con ganancias retenidas. Aunque, sus ganancias retenidas son inferiores a la cantidad de emisión, las compañías deberían considerar financiar una porción con ganancias retenidas.

Tabla 14. Cantidad de emisión-compañías que procesan alimentos

En millones de dólares (USD)

\begin{tabular}{ccc} 
En millones de dólares (USD) & \\
Compañía & $\begin{array}{c}\text { Monto de } \\
\text { emisión }\end{array}$ & $\begin{array}{c}\text { Ganancias } \\
\text { acumuladas }\end{array}$ \\
\hline Industrias Toni & 227.05 & 6.11 \\
Heladosa & 2.50 & 0.69 \\
Floralp & 2.50 & 0.29 \\
Industria lojana & 5.00 & 1.41 \\
Universal sweet & 10.00 & 1.30 \\
Sumesa & 7.00 & 2.79 \\
Alimentsa & 3.00 & 4.04 \\
Moderna & 8.00 & 23.45 \\
\hline
\end{tabular}

Tabla 15. Cantidad de emisión-compañías de productos básicos

En millones de dólares (USD)

\begin{tabular}{lcc} 
Compañía & $\begin{array}{c}\text { Monto de } \\
\text { emisión }\end{array}$ & $\begin{array}{c}\text { Ganancias } \\
\text { acumuladas }\end{array}$ \\
$\begin{array}{l}\text { Azucarera } \\
\text { Valdéz }\end{array}$ & 10.00 & 34.00 \\
Cofina & 3.00 & 0.69 \\
Labiza & 1.00 & 1.00 \\
\hline
\end{tabular}

Todas las empresas, excepto Industrias Toni, emitieron deuda. El gasto por emisión representó alrededor del $2 \%$ de la emisión total.

En este caso particular, es aconsejable que la compañía Azucarera Valdéz financie sus inversiones con utilidades retenidas con el fin de ahorrar dinero y como analizamos anteriormente, la empresa está muy endeudada.

\section{Conclusiones}

Se encontró que las empresas estudiadas eligieron su estructura de capital con base en la política de la Bolsa Valores de Guayaquil y su propia política. Sin embargo, los gerentes deben considerar ambas teorías para maximizar el valor de la empresa y reducir los costos. Se ha demostrado a través de estas teorías que las decisiones sobre la estructura del capital influyen en el valor global del negocio.

En relación con la teoría del intercambio, se encontró la estructura de capital óptima. Las empresas que procesan alimentos tienden a ser sub-endeudadas. La mayoría de éstas se acercan a la estructura de capital óptima. Mientras que las empresas de productos básicos están sobre-endeudadas, las cuales deben reducir su estructura de capital para evitar problemas financieros. La gran mayoría de las empresas de procesamiento de alimentos tienden a tener una tasa de cobertura más alta (promedio: 8,86 ) que las empresas de productos básicos (promedio: $2,16)$. Esto implica que las empresas rentables pueden aumentar el coeficiente de deuda ya que tienen la capacidad de pagar intereses a los tenedores de deuda.

En relación con la teoría del orden jerárquico, las empresas estudiadas han mantenido sus ganancias. Aunque las utilidades retenidas no cubren el total de sus necesidades, deben considerarlo en primer lugar para financiar sus activos para reducir costos. Luego, la teoría sigue porque la mayoría de las empresas emitieron deuda. 
Sería interesante analizar otros sectores como: servicios y otros segmentos de las industrias en el mercado ecuatoriano para contrastar los resultados obtenidos de este trabajo. Como resultado, los gerentes podrían ser conscientes de que hay teorías que podrían ayudarles a maximizar el valor de la empresa, minimizar el costo del capital y reducir los costos.

\section{Bibliografía}

[1] Jensen, N. S., \& Uhl, F. T. (August de 2008). Capital Structure in European SMEs. Obtenido de http://pure.au.dk/ portal/files/3244/Capital_Structure_in_Eur opean_SMEs.pdf

[2] Chirinko, R., \& Singha, A. (2000). Testing static trade-off against pecking order models of capital structure: A critical comment. Journal of Financial Economics, 417-425.

[3] Frank, M., \& Goyal, V. (2003). Testing the pecking order theory of capital structure. Journal of Financial Economics, 217-248.

[4] Leary, M., \& Roberts, M. (2010). The Pecking order, debt capacity, and information asymmetry. Journal of Financial Economics, 332-355.

[5] Shyam-Sunder, L., \& Myers, S. (1999). Testing static tradeoff against pecking order models of capital structure. Journal of Financial Economics, 219-244.

[6] Lemmon, M. L., \& Zender, J. (2010). Debt capacity and tests of capital structure. Journal of Financial and Quantitative analysis, 1161-1187.

[7] Miglo, A. (2014). Capital Structure of Internet Companies. Journal of Internet Commerce, pp.
253-281.

[8] Maldonado, F., \& Proaño, G. (2015). La Industria en Ecuador. EKOSNEGOCIOS.COM, 46-58.

[9] Brealey, Myers, Allen . (2011). PRINCIPLES OF CORPORATE FINANCE. New York: McGraw-Hill.

[10]Brealey, Myers, Allen. (2011). Principles of Corporate Finane. In Principles of Corporate Finance (p. 447). McGraw-Hill Irwin.

[11]Martin, H. Kent Baker and Gerald S. (2011). CAPITAL STRUCTURE AND CORPORATE FINANCING DECISIONS: theory, evidence and practice. New Jersey, USA: John Wiley \& Sons, Inc.

[12] Grinblatt-Titman. (2002). Financial Markets and Corporate Strategy. The McGraw-Hill.

[13]Banco Mundial. (2017). Banco Mundial. Obtenido de Banco Mundial: https://datos.bancomundial.org/pais/ecuad or

[14] Asamblea Nacional Ecuador. (2010). Código Orgánico de la Producción, Comercio e Inversiones., (pág. 56). Quito.

[15] Damodaran, A. (n.d.). Finding the Right Financing Mix: The capital Structure Decision. Stern School of Business.

[16] Aswath Damodaran. (s.f.). Damodaran Online. Obtenido de Damodaran Online: http://pages.stern.nyu.edu/ adamodar/Ne w_Home_Page/valquestions/syntrating.ht $\mathrm{m}$

[17] Consejo Nacional de Valores. (2015). Codificación de resoluciones del Consejo Nacional de Valores. 